\title{
(6) OPEN ACCESS \\ Cigarette stick as valuable communicative real estate: a content analysis of cigarettes from 14 low-income and middle-income countries
}

\author{
Katherine C Smith, ${ }^{1,2}$ Carmen Washington, ${ }^{2}$ Kevin Welding, ${ }^{2}$ Laura Kroart, ${ }^{2}$ \\ Adami Osho, ${ }^{2}$ Joanna E Cohen ${ }^{1,2}$
}

${ }^{1}$ Department of Health, Behavior and Society, Johns Hopkins Bloomberg School of Public Health, Baltimore, Maryland, USA

${ }^{2}$ Institute for Global Tobacco Control, Johns Hopkins Bloomberg School of Public Health, Baltimore, Maryland USA

\section{Correspondence to} Dr Katherine Clegg Smith, Department of Health, Behavior and Society, Johns Hopkins Bloomberg School of Public Health, 624 N. Broadway, Baltimore MD 21205, USA; katecsmith@jhu.edu

Received 2 May 2016 Revised 10 July 2016 Accepted 27 July 2016 Published Online First 17 August 2016

\section{CrossMark}

To cite: Smith $\mathrm{KC}$ Washington C, Welding K, et al. Tob Control 2017;26:604-607.

\section{ABSTRACT}

Background The current cigarette market is heavily focused on low-income and middle-income countries. Branding of tobacco products is key to establishing and maintaining a customer base. Greater restrictions on marketing and advertising of tobacco products create an incentive for companies to focus more on branding via the product itself. We consider how tobacco sticks are used for communicative purposes in 14 low-income and middle-income countries with extensive tobacco markets. Methods In 2013, we collected and coded 3232 cigarette and kretek packs that were purchased from vendors in diverse neighbourhoods in 44 cities across the 14 low-income and middle-income countries with the greatest number of smokers. A single stick from each pack was assessed for branding, decorative and communicative elements using a common coding framework. Stick communication variables included brand name, brand image/logo, brand descriptor, colour and design carried through from pack, 'capsule cigarette' symbol, and embellishment of filter end.

Results Communication and branding on the stick is essentially ubiquitous (99.75\%); $97 \%$ of sticks include explicit branding (brand name or logo present). Colour is commonly carried through from the pack $(95 \%)$, and some sticks (13\%) include decorative elements matching the pack. Decorative elements can be found anywhere on the stick, including the filter tip (8\%), and 'convertible' cigarettes include a symbol to show where to push.

Conclusions Cigarette sticks are clearly valuable 'real estate' that tobacco companies are using for communicative purposes. Across all countries and brands, the stick communicates branding via text, colour and imagery.

\section{INTRODUCTION}

The burden of tobacco use has shifted away from high-resource countries and is now borne most heavily by those living in low-income and middle-income countries. ${ }^{1}$ The Framework Convention on Tobacco Control (FCTC) Article 13 calls for greater restrictions on marketing and advertising of tobacco products, including efforts to promote brand image and appeal. As a consequence, opportunities to promote tobacco products via traditional channels are increasingly restricted around the globe. Companies have an incentive to focus greater attention on packaging and the cigarette itself to convey brand image. ${ }^{2}$ Ayo-Yusuf and Agaku $^{4}$ describe the cigarette itself as the 'primary' level of packaging, and argue that enhancements in cigarette packaging are associated with increased sales and also influence sensory perceptions around cigarettes in ways that are key to smoking behaviour and addiction.

The communicative potential of the product itself is considerable; Hammond ${ }^{5}$ estimated that a pack-a-day smoker would be exposed to a tobacco pack 7000 times per year. Following this logic, using a conservative estimate of 10 puffs per cigarette, the same pack-a-day (20 cigarettes) smoker would encounter a cigarette $\sim 70000$ times per year. The nature of the engagement is also elevated; whereas smokers display the pack, they actually consume the stick. ${ }^{6}$ Borland and Savvas (p. 335) ${ }^{7}$ state that "cigarette stick shape, color and design are differentiable to smokers on qualities, such as attractiveness, perceived quality and estimated strength of taste." There is a small but growing literature demonstrating ways in which the appearance of the stick has the capacity to act as a form of promotion, and can facilitate brand differentiation and impact smokers' perceptions of specific brands' attributes. $^{7-11}$

We consider ways in which the sticks are being used for communicative purposes in 14 low-income and middle-income countries with extensive tobacco markets. We examine the extent to which the cigarette stick is used to communicate information and the type of information being communicated via the stick.

\section{METHODS}

Our analysis of stick communicative variability is based on systematic surveillance of cigarettes available for purchase in 2013. We collected data in the 14 low-income and middle-income countries with the greatest number of smokers (Bangladesh, Brazil, China, Egypt, India, Indonesia, Mexico, Pakistan, Philippines, Russian Federation, Thailand, Turkey, Ukraine and Vietnam). In each country, we collaborated with a local agency to implement a common data collection protocol. ${ }^{12}$ Our sampling approach maximised the number and diversity of packs acquired in each country. We collected data from 44 cities, beginning in the country's most populated city and selecting two additional cities from the next nine most populated on the basis of cultural, geographic, religious and linguistic factors. In China we sampled five cities. In each city, we purchased packs from vendors in high-income, medium-income and low-income neighbourhoods.

In total, we collected and coded 3232 cigarette and kretek packs. We assessed each pack and a 
single stick from within for branding, decorative and communicative elements. We developed a coding framework that was extensively tested before being independently applied by two trained coders. We created seven variables pertaining to branding and other communication on the stick:

- Brand name on stick;

- Brand image or logo on stick;

- Brand descriptor language on stick (eg, 'light' or 'silver');

- Colour carried through on stick from pack;

- Design carried through on stick from pack;

- 'Capsule cigarette' symbol or indicator;

- Embellishment of filter end (eg, heart cut out or coloured dot).

All coding discrepancies were reconciled by a third trained reviewer. To assess data reliability, Cohen's $\kappa$, prevalence adjusted $\kappa$ (PABAK) and interclass correlation coefficient were calculated as appropriate. For the seven variables included in this analysis, the average per cent agreement was 95\%, average $\kappa$ was 0.76 and the average PABAK was 0.9 .
RESULTS

Almost every stick in our sample $(99.75 \%, \mathrm{n}=3225)$ included some communicative or branding element (figure 1A). Brand name is included on 91\% $(n=2936)$ of sticks (figure 1B) and $32 \%(n=1046)$ included some pictorial depiction of the brand logo (figure 1B), such that $97 \%(n=3146)$ of sticks included one or other branding element. The colour palette from the outside of the pack was commonly carried through to the stick (figure 1C); 95\% $(n=3080)$ of sticks included colour elements that matched the pack. Beyond colour, some sticks (13\%, $\mathrm{n}=413$ ) carried decorative design elements (including patterns and imagery) through to the stick filter and paper barrel (figure 1C).

We found decorative elements on all aspects of the stick (except the end to be lit). Filter tips were decorative on $8 \%$ $(n=224)$ of all sticks (figure 1D). Decorations on filter tips included coloured dots (in various colours), printed images such as smiley faces and circles, and decorative cut outs (hearts, stars, profile images) that were both coloured and plain. Finally, sticks
A

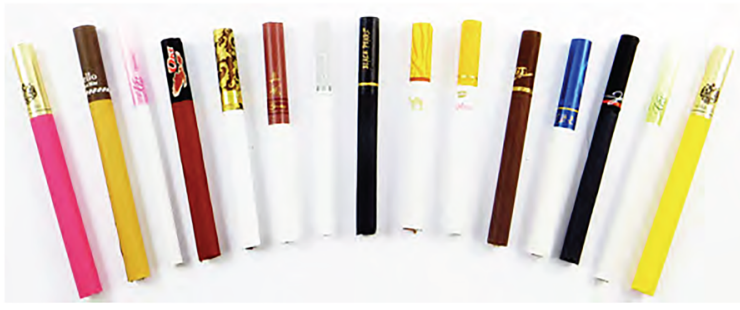

C

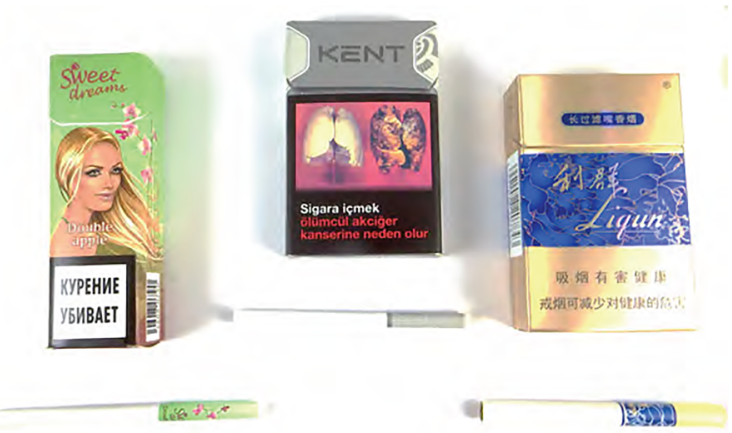

D

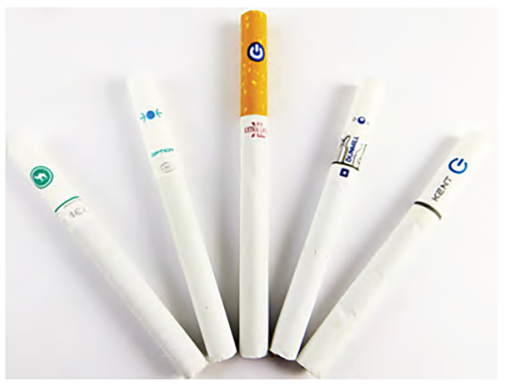

B
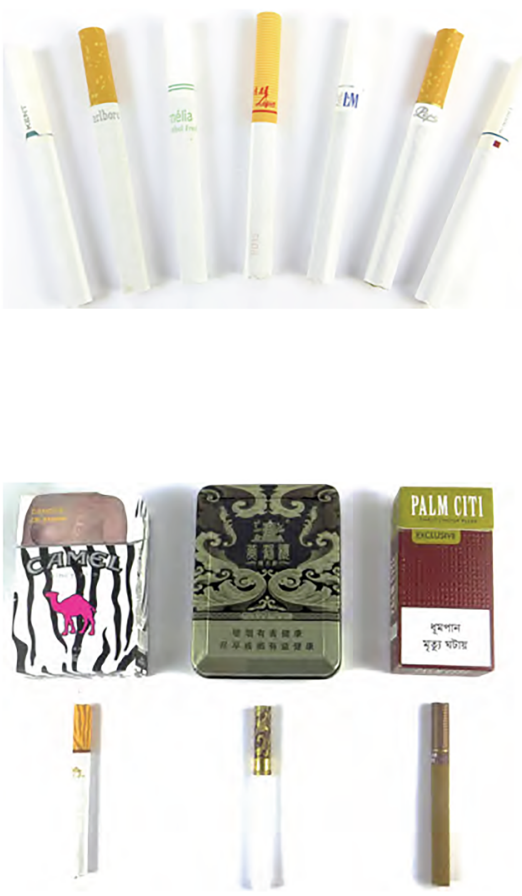

E

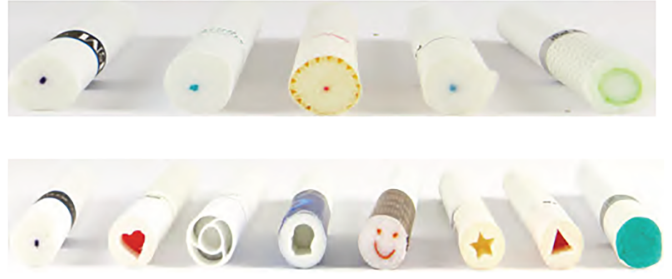

Figure 1 Examples of communicative elements on the stick. (A) Communicative elements. (B) Branding text and logos. (C) Colour and design carried through from pack. (D) Capsule indicators. (E) Decorative filter tips. 
were used to convey information about use of the product, particularly in relation to capsule technology (figure 1E); 83 sticks $(2.6 \%)$ included an indicator of capsule technology, including where to push on the stick to initiate the product change.

\section{DISCUSSION}

To the best of our knowledge, this is the first systematic collection and analysis of cigarette sticks from multiple countries. This analysis demonstrates that use of the stick for communicative purposes is a ubiquitous practice in these 14 countries. Every element of the stick-from the paper to the filter barrel to its tip-has potential communicative utility. Thus, policies that take into consideration the communicative potential of the stick as well as the pack are important tools for effective control of cigarette marketing and promotion.

Wakefield et $a l^{13}$ classified cigarettes as a 'badge product', such that much of their value pertains to witnessed consumption, and embodiment and demonstration of brand sentiment on the part of the user. Limiting cigarette branding is important to constrain tobacco companies' ability to create a sense of connection and identification with a particular product, as well as conveying an enhanced sense of self through consumption of the product, and ultimately referencing brand imagery to affirm one's decision to smoke. ${ }^{14}{ }^{15}$ These data demonstrate a need to attend to the stick within efforts to advance policy to limit the impact of cigarette branding.

In addition to brand names and logos, sticks in our sample contained colour elements that referenced and reinforced colours on the pack (eg, silver, red, blue). Colour is important for brand identity, ${ }^{16}$ and for conveyance of product characteristics such as strength and the deceptive notion of less harm'. ${ }^{6} 1617$ Our study demonstrates how communication through colour-coding does not end when one opens the pack; the cigarettes often match the coloured messaging being conveyed on the exterior.

Cigarettes with decorative cut-outs and patterns on the filter tips are potentially whimsical and cute; gold bands may convey luxury; and bright and pastel colours and patterns on paper and filters may create a sense of an overtly feminine product. ${ }^{18}$

In line with previous studies, ${ }^{7-11}$ we demonstrate that cigarette sticks are valuable 'real estate' that can be used for communicative purposes. Currently, this space is dominated by industry messaging for branding or some other form of product appeal messaging. Borland and Savvas ${ }^{7}$ noted the prevalence of branding and decorative elements on cigarette sticks in Australia (before implementation of plain packaging legislation) and argued that the cost of such efforts means that this must have perceived value to cigarette companies. The value of this real estate presumably increases in contexts where cigarettes are purchased as singles, as in these instances, the personal connection with and consumption of the brand will be bereft of the messaging that is usually conveyed via the pack. Regardless of whether cigarettes are purchased in packs or singly, we might usefully think of sticks as tiny billboards that currently convey tobacco company messages. Borland and Savvas ${ }^{7}$ argued that brand loyalty might be somewhat diminished if sticks were standardised and differentiation by the stick was no longer possible. Australian plain packaging legislation appropriately extends restrictions and specifications to the stick itself. The stick must be plain white and with either a white or 'imitation cork' paper to cover the filter; there can be no branding of any kind on the stick. ${ }^{7}$

We did not measure the sticks, and thus we cannot incorporate differences in cigarette length in this analysis. Stick size (length and diameter) has previously been shown to communicate messages of cigarette attributes and reduced harm $^{89}$ and establishing the variation in stick size in different countries could be an important aspect of future work.

Making the stick plain and standardised may not, however, be the 'end-goal' for tobacco control; cigarette sticks could also be made deliberately unattractive, ${ }^{19}$ so as to disrupt the brand identification with an otherwise appealing product. ${ }^{8-10}$ Moreover, Hassan and Shiu ${ }^{20}$ point out that other consumer products carry health warnings on the packaging and the item itself, and provide data that suggest that including a dissuasive on the cigarette itself may serve to prompt quit intentions. Dissuasive messaging can take the form of making the cigarette itself less attractive ${ }^{19}$ or display a health message ${ }^{20}$ or explicit warning. ${ }^{9}$ FCTC Article 11 recommends communication policies that extend beyond the pack to the stick itself. It may be possible for marketing on the stick to be limited and for the 'real estate' on the stick to be used instead for targeted and effective health messaging.

\section{What this paper adds}

- The cigarette itself is a potentially powerful canvas for messaging about tobacco. As of now, this space is being used exclusively by tobacco companies.

- Branding on the stick is almost ubiquitous on cigarettes purchased in the 14 low- and middle-income countries with the greatest number of smokers.

- A goal for tobacco control policy could be for marketing on the stick to be prohibited and for the 'real estate' on the stick to be used instead for targeted and effective health messaging.

Funding Bloomberg Initiative to Reduce Tobacco Use.

Competing interests None declared.

Provenance and peer review Not commissioned; externally peer reviewed.

Open Access This is an Open Access article distributed in accordance with the Creative Commons Attribution Non Commercial (CC BY-NC 4.0) license, which permits others to distribute, remix, adapt, build upon this work non-commercially, and license their derivative works on different terms, provided the original work is properly cited and the use is non-commercial. See: http://creativecommons.org/ licenses/by-nc/4.0/

\section{REFERENCES}

1 Jha P, Peto R. Global effects of smoking, of quitting and of taxing tobacco. N Engl J Med 2014;370:60-8.

2 Hammond D, Daniel S, White C. The effect of branding and plain packaging on youth in the United Kingdom. J Adolescent Health 2013;52:151-7.

3 Moodie C, Angus K, Ford A. The importance of cigarette packaging in a 'dark' market: the 'Silk Cut' experience. Tob Control 2014;23:274-8.

4 Ayo-Yusuf OA, Agaku IT. The association between smokers' perceived importance of the appearance of cigarettes/cigarette packs and smoking sensory experience: a structural equation model. Nicotine Tob Res 2015;17:91-7.

5 Hammond D. Health warning messages on tobacco products: a review. Tob Control 2011;20:327-37

6 Hoek J, Gendall P, Eckert C, et al. Dissuasive cigarette sticks: the next step in standardised ('plain') packaging? Tob Control 2015;0:1-7.

7 Borland R, Savvas $S$. Effects of stick design features on perceptions of characteristics of cigarettes. Tob Control 2013;22:331-7.

8 Ford A, Moodie C, MacKintosh AM, et al. Adolescent perceptions of cigarette appearance. Eur J Public Health 2014;24:464-8.

9 Moodie C, Ford A, MacKintosh A, et al. Are all cigarettes just the same? Female's perceptions of slim, coloured, aromatised and capsule cigarettes. Health Educ Res 2014;30:1-12. 
10 Moodie C, Ford A, MacKintosh A, et al. Novel means of using cigarette packaging and cigarettes to communicate health risk and cessation messages: a qualitative study. Int J Ment Health Addict 2015;13:333-44.

11 Thrasher JF, Abad-Vivero EN, Moodie C, et al. Cigarette brands with flavour capsules in the filter: trends in use and brand perceptions among smokers in the USA, Mexico and Australia, 2012-2014. Tob Control 2016:25:275-83.

12 Smith KC, Washington C, Brown J, et al. The Tobacco Pack Surveillance System (TPackSS): a protocol for assessing health warning compliance, design features and appeals of tobacco packs sold in low-and middle-income countries. J Med Internet Res Public Health Surveill 2015;1:e8.

13 Wakefield M, Morley C, Horan JK, et al. The cigarette pack as image: new evidence from tobacco industry documents. Tob Control 2002;11(Suppl 1):i73-80.

14 Hastings G, MacFadyen L. Smoking, branding, and the meaning of life. Tob Control 1998;7:107-8.
15 Cook BL, Wayne GF, Keithly L, et al. One size does not fit all: how the tobacco industry has altered cigarette design to target consumer groups with specific psychological and psychosocial needs. Addiction 2003;98:1547-61.

16 Moodie C, Ford A, Mackintosh AM, et al. Young people's perceptions of cigarette packaging and plain packaging: an online survey. Nicotine Tob Res 2012;14: 98-105.

17 Bansal-Travers M, O'Connor R, Fix BV, et al. What do cigarette pack colors communicate to smokers in the U.S.? Am J Prev Med 2011:40:683-9.

18 Carpenter CM, Wayne GF, Connolly GN. Designing cigarettes for women: new findings from the tobacco industry documents. Addiction 2005;100:837-51.

19 Hoek J, Robertson C. How do young adult female smokers interpret dissuasive cigarette sticks? A qualitative analysis. J Soc Mark 2015:5:21-39.

20 Hassan LM, Shiu E. No place to hide: two pilot studies assessing the effectiveness of adding a health warning to the cigarette stick. Tob Control 2015;24:e3-5. 